\title{
Similarity Solutions of MHD Mixed Convection Flow with Variable Reactive Index, Magnetic Field, and Velocity Slip Near a Moving Horizontal Plate: A Group Theory Approach
}

\author{
W. A. Khan, ${ }^{1}$ Md. Jashim Uddin, ${ }^{2,3}$ and A. I. Md. Ismail ${ }^{2}$ \\ ${ }^{1}$ Department of Engineering Sciences, PN Engineering College, \\ National University of Sciences E Technology, Karachi 75350, Pakistan \\ ${ }^{2}$ School of Mathematical Sciences, Universiti Sains Malaysia, Penang 11800, Malaysia \\ ${ }^{3}$ American International University-Bangladesh, Banani, Dhaka 1213, Bangladesh \\ Correspondence should be addressed to Md. Jashim Uddin, jashim_74@yahoo.com
}

Received 9 February 2012; Accepted 7 August 2012

Academic Editor: Eihab M. Abdel-Rahman

Copyright $@ 2012$ W. A. Khan et al. This is an open access article distributed under the Creative Commons Attribution License, which permits unrestricted use, distribution, and reproduction in any medium, provided the original work is properly cited.

\begin{abstract}
The mixed convection of Newtonian fluid flow along a moving horizontal plate with higher-order chemical reaction, variable concentration reactant, and variable wall temperature and concentration is considered. Velocity slip and the thermal convective boundary conditions are applied at the plate surface. The governing partial differential equations are transformed into similarity equations via dimensionless similarity transformations developed by one-parameter continuous group method. The numerical solutions of the transformed ordinary differential equations are constructed for velocity, temperature and concentration functions, the skin friction factor, the rate of heat, and the rate of mass transfer using an implicit finite difference numerical technique. The investigated parameters are buoyancy parameters $\lambda_{1}, \lambda_{2}$, chemical reaction parameter $K$, suction/ injection parameter $f w$, velocity slip parameter $a$ convective heat transfer parameter $\gamma$, magnetic parameter $M$, Prandtl number Pr and Schmidt number, Sc. Comparison with results from the open literature shows a very good agreement.
\end{abstract}

\section{Introduction}

A convection situation involving both free and forced convection is known as mixed convection and has been an important topic because of its application in electronic equipment cooled by a fan and flows in the ocean and in the atmosphere $([1,2])$. In mixed convection flows, the forced and the free convection effects are of comparable magnitude. Thus, mixed convection occurs if the effect of buoyancy forces on a forced flow or vice versa is significant. The laminar mixed convection takes place in various applications in thermal engineering 
and science and has drawn attention from researchers in the past decades. Examples of these applications include atmospheric boundary layer flows, solar energy systems, boilers, compact heat exchangers, and cooling of electronic devices [3,4]. Many studies exist for the mixed convection boundary layer flow about vertical/inclined/horizontal plate and wedge surfaces immersed in a viscous fluid. The details of mixed convection can be found in books by Chen and Armaly [5], Jaluria [6], Gebhart et al. [7], Bejan [8], Pop and Ingham [9] and in the articles in the literatures by Lloyd and Sparrow [10], Schneider [11], Wilks [12], Yao [13], Ramachandran et al. [14], Moulic and Yao, [15], Daskalakis et al. [16], Fan et al. [17], Magyari et al. [18], Hassanien et al. [19], Aydin and Kaya [20], Ishak [21], Moulic and Yao [22], Datta et al. [23], Ishak et al. [24], Magyari [25].

In all these studies, conventional no slip boundary conditions were applied at the surface. However, fluid flows in micro/nanoscale such as micronozzles, micropumps, microturbines, microheat exchangers, microvalves, turbines, sensors, and actuators are important for micro and nanoscience and the conventional no slip boundary condition at the solid fluid interface must be replaced with the slip condition [26]. The slip condition states that the tangential component of the velocity at the surface is equal to the velocity gradient normal to the surface $([27,28])$. Slips may occur on a stationary/moving boundary when the fluid is in particulate form such as in the form of emulsions, suspensions, foams, and polymer solutions $[29,30]$. An excellent review for the fundamentals, theory, and applications of these microscale systems may be found in the text by Duncan and Peterson [31] Gad-el-Hak [32]. Slips effect on flow field has been studied by various authors; Fang and Lee [33], Martin and Boyd [34], Matthews and Hill [35], Wang [36]. Cao and Baker [37] studied laminar mixed convection over an isothermal vertical plate with first-order momentum and thermal discontinuities at the wall. The hydrodynamic and thermal slip flow boundary layer over a flat plate with constant heat flux boundary condition has been investigated by Hak [27] who concluded that as the slip parameter increases, the slip velocity increases and the wall shear stress decreases.

Due to the nonlinearities of the governing equations, no analytical solution is available and the nonlinear equations usually solved numerically subject to initial/boundary conditions. The solution of differential equations with/without initial/boundary conditions is presented by different authors such as Ames [38], Boyce [39], Na [40], Zauderer [41], Seshadri and $\mathrm{Na}$ [42], Kincaid and Cheney [43], Strauss [44], Aziz [45], and White and Subramanian [46].

We study combined convective Newtonian fluid flow adjacent to a moving horizontal permeable flat plate with higher order chemical reaction taking into account velocity slip and convective surface boundary conditions. The governing boundary layer equations are converted into a two point boundary value problem using similarity transformations developed by group method and the transformed equations are solved by means of an implicit finite difference numerical method. Graphs are plotted for velocity, temperature and concentration profiles as well as the skin friction factor, the rate of heat transfer and the rate of mass transfer to exhibits the influences of the parameters involving in our problem.

\section{Mathematical Formulations of the Problem}

Consider a continuous moving permeable horizontal flat plate which moves with a nonuniform velocity $U(x)$ as shown in Figure 1. A variable magnetic field of strength $B(x)$ is applied to the normal direction of the plate. The bottom surface of the plate is heated by convection from a hot fluid of temperature $T_{f}(x)$ which provides a heat transfer 


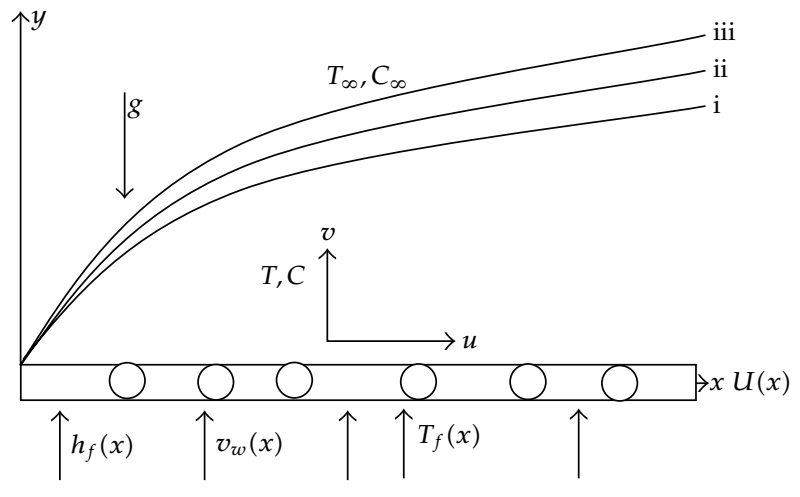

(i) Concentration,
(ii) Thermal and
(iii) Momentum B.L

Figure 1: Physical configuration and coordinate system the problem.

coefficient $h_{f}(x)$. The viscous dissipation in the energy equation is neglected. Assume that fluid properties are constant accepts in buoyancy term. We will further assume the magnetic Reynolds number; the electric field owing to the polarization of charges and Hall effects are negligible. Under the forgoing assumptions the governing equations in dimensional form can be written as $[47,48]$

$$
\begin{gathered}
\frac{\partial u}{\partial x}+\frac{\partial v}{\partial y}=0 \\
u \frac{\partial u}{\partial x}+v \frac{\partial u}{\partial y}=-\frac{1}{\rho} \frac{\partial p}{\partial x}+v \frac{\partial^{2} u}{\partial y^{2}}-\frac{\sigma B^{2}(x)}{\rho} u \\
-\frac{1}{\rho} \frac{\partial p}{\partial y}+g \beta_{T}\left(T-T_{\infty}\right)+g \beta_{C}\left(C-C_{\infty}\right)=0 \\
u \frac{\partial T}{\partial x}+v \frac{\partial T}{\partial y}=\alpha \frac{\partial^{2} T}{\partial y^{2}} \\
u \frac{\partial C}{\partial x}+v \frac{\partial C}{\partial y}=D \frac{\partial^{2} C}{\partial y^{2}}-k_{0}\left(C-C_{\infty}\right)^{n} .
\end{gathered}
$$

The boundary conditions are [49]

$$
\begin{gathered}
u=U(x)+N_{1}(x) v \frac{\partial u}{\partial y}, \quad v=-v_{w}(x), \quad-k \frac{\partial T}{\partial y}=h_{f}(x)\left[T_{f}(x)-T_{w}\right], \\
C=C_{w}(x) \quad \text { at } y=0, \\
u \longrightarrow 0, \quad v \longrightarrow 0, \quad T \longrightarrow T_{\infty}, \quad C \longrightarrow C_{\infty} \quad \text { as } y \longrightarrow \infty,
\end{gathered}
$$


where $v_{w}(x)$ : velocity normal to the plate, $v$ : coefficient of kinematic viscosity, $\rho$ : density of the fluid, $\sigma$ : electric conductivity, $p$ : pressure, $k$ : thermal conductivity, $\alpha$ : thermal diffusivity, $D$ : mass diffusivity of species in fluid, $\beta_{T}$ : volumetric thermal coefficient, $\beta_{C}$ : volumetric concentration coefficient, $g$ : acceleration due to gravity, $k_{0}$ : reaction rate, $n$ : order of chemical reaction, and $N_{1}(x)$ : velocity slip factor with dimension (velocity) ${ }^{-1}$.

Introducing stream function $\psi$, dimensionless temperature function $\theta$ and concentration function $\phi$ are defined by

$$
u=\frac{\partial \psi}{\partial y}, \quad v=-\frac{\partial \psi}{\partial x}, \quad \theta=\frac{T-T_{\infty}}{T_{f}-T_{\infty}}, \quad \phi=\frac{C-C_{\infty}}{C_{w}-C_{\infty}}
$$

Using (2.3) we get from (2.1)

$$
\begin{gathered}
\frac{\partial \psi}{\partial y} \frac{\partial^{2} \psi}{\partial x \partial y}-\frac{\partial \psi}{\partial x} \frac{\partial^{2} \psi}{\partial y^{2}}=-\frac{1}{\rho} \frac{\partial p}{\partial x}+\nu \frac{\partial^{3} \psi}{\partial y^{3}}-\frac{\sigma B^{2}(x)}{\rho} \frac{\partial \psi}{\partial y} \\
-\frac{1}{\rho} \frac{\partial p}{\partial y}+g \beta_{T} \theta \Delta T+\beta_{C} \phi \Delta C=0 \\
\frac{\partial \psi}{\partial y} \frac{\partial}{\partial x}(\theta \Delta T)-\frac{\partial \psi}{\partial x} \frac{\partial}{\partial y}(\theta \Delta T)=\alpha \frac{\partial^{2}}{\partial y^{2}}(\theta \Delta T) \\
\frac{\partial \psi}{\partial y} \frac{\partial}{\partial x}(\phi \Delta C)-\frac{\partial \psi}{\partial x} \frac{\partial}{\partial y}(\phi \Delta C)=D \frac{\partial^{2}}{\partial y^{2}}(\phi \Delta C)-k_{0}(\phi \Delta C)^{n}
\end{gathered}
$$

Eliminating the pressure gradient terms from (2.4) and (2.5) by cross-differentiation, we get

$$
\begin{gathered}
\nu \frac{\partial^{4} \psi}{\partial y^{4}}+\frac{\partial \psi}{\partial x} \frac{\partial^{3} \psi}{\partial y^{3}}-\frac{\partial \psi}{\partial y} \frac{\partial^{3} \psi}{\partial x \partial y^{2}}-g \beta_{T}(\Delta T \theta)_{x}-g \beta_{T}(\Delta C \phi)_{x}-\frac{\sigma B^{2}(x)}{\rho} \psi_{y y}=0 \\
\alpha \frac{\partial^{2}}{\partial y^{2}}(\theta \Delta T)+\frac{\partial \psi}{\partial x} \frac{\partial}{\partial y}(\theta \Delta T)-\frac{\partial \psi}{\partial y} \frac{\partial}{\partial x}(\theta \Delta T)=0 \\
D \frac{\partial^{2}}{\partial y^{2}}(\phi \Delta C)+\frac{\partial \psi}{\partial x} \frac{\partial}{\partial y}(\phi \Delta C)-\frac{\partial \psi}{\partial y} \frac{\partial}{\partial x}(\phi \Delta C)-k_{0}(\phi \Delta C)^{n}=0
\end{gathered}
$$

The boundary conditions (2.2) become

$$
\begin{gathered}
\frac{\partial \psi}{\partial y}=U(x)+v N_{1}(x) \frac{\partial^{2} \psi}{\partial y^{2}}, \quad-\frac{\partial \psi}{\partial x}=v_{w}(x) \frac{\partial \theta}{\partial y}=-\frac{h_{f}(x)}{k}[1-\theta(0)], \quad \phi=1 \quad \text { at } y=0 \\
\frac{\partial \psi}{\partial y} \longrightarrow 0, \quad \frac{\partial \psi}{\partial x} \longrightarrow 0, \quad \theta \longrightarrow 0, \quad \phi \longrightarrow 0 \quad \text { as } y \longrightarrow \infty
\end{gathered}
$$




\section{Application of Group Theory}

We will now search group invariant solutions (similarity solutions) of (2.8) to (2.9) under a particular continuous one-parameter group. We define the following one-parameter group transformations $\Gamma$ :

$$
\begin{gathered}
x^{*}=e^{\varepsilon \alpha_{1}} x, \quad y^{*}=e^{\varepsilon \alpha_{2}} y, \quad \psi^{*}=e^{\varepsilon \alpha_{3}} \psi, \quad \theta^{*}=e^{\varepsilon \alpha_{4}} \theta, \quad \phi^{*}=e^{\varepsilon \alpha_{5}} \phi, \quad B^{*}=e^{\varepsilon \alpha_{6} B} \\
\Delta T^{*}=e^{\varepsilon \alpha_{7}} \Delta T, \quad \Delta C^{*}=e^{\varepsilon \alpha_{8}} \Delta C, \quad U=e^{\varepsilon \alpha_{9}} U, \quad v_{w}^{*}=e^{\varepsilon \alpha_{10}} v_{w}, \quad h_{f}^{*}=e^{\varepsilon \alpha_{11}} h_{f}
\end{gathered}
$$

Here $\varepsilon$ is the parameter of the group and $\alpha_{i}(i=1,2,3, \ldots, 11)$ are the arbitrary real numbers whose interrelationship will be determined by our investigation. Transformations in (3.1) may be considered as point transformations which transform the coordinates

$$
\left(x, y, \psi, \theta, \phi, B, \Delta T, \Delta C, U, v_{w}, h_{f}\right) \text { to }\left(x^{*}, y^{*}, \psi^{*}, \theta^{*}, \phi^{*}, B^{*}, \Delta T^{*}, \Delta C^{*}, U^{*}, v_{w}^{*}, h_{f}^{*}\right) .
$$

We now find the relationship among the exponents $\alpha_{i}$ such that (2.8) with the boundary conditions in (2.9) are invariant (the structure of the equations remains same before and after the transformations) under the transformation group in (3.1). Substituting transformations in (3.1) into (2.8) and equating various exponents of $e$ for constant conformally invariant, we get the following algebraic equations:

$$
\begin{gathered}
4 \alpha_{2}-\alpha_{3}=\alpha_{1}+3 \alpha_{2}-2 \alpha_{3}=\alpha_{1}-\alpha_{4}-\alpha_{7}=\alpha_{1}-\alpha_{5}-\alpha_{8}=2 \alpha_{2}-\alpha_{3}-2 \alpha_{6}, \\
2 \alpha_{2}-\alpha_{4}-\alpha_{7}=\alpha_{1}+\alpha_{2}-\alpha_{3}-\alpha_{4}-\alpha_{7}, \\
2 \alpha_{2}-\alpha_{5}-\alpha_{8}=\alpha_{1}+\alpha_{2}-\alpha_{3}-\alpha_{5}-\alpha_{8}=n\left(-\alpha_{5}-\alpha_{8}\right) .
\end{gathered}
$$

Using (3.1) in boundary conditions (2.9) and equating various exponents of $e$, we get

$$
\alpha_{2}-\alpha_{3}=-\alpha_{9}, \quad \alpha_{1}-\alpha_{3}=-\alpha_{10}, \quad \alpha_{2}=-\alpha_{11} .
$$

Without loss of generality, we may put $\alpha_{4}=\alpha_{5}=0$.

Solving (3.3) and (3.4) we have the following relationship among the exponents

$$
\begin{gathered}
\alpha_{1}=\frac{(7-5 n)}{4 \alpha_{8}}, \quad \alpha_{2}=\frac{(1-n)}{2 \alpha_{8}}, \quad \alpha_{3}=\frac{(5-3 n)}{4 \alpha_{8}}, \quad \alpha_{4}=0, \quad \alpha_{5}=0, \\
\alpha_{7}=\alpha_{8}, \quad \alpha_{9}=\frac{(3-n)}{4 \alpha_{8}}, \quad \alpha_{6}=\alpha_{10}=\alpha_{11}=\frac{(n-1)}{2 \alpha_{8}} .
\end{gathered}
$$

It can be easily verified that (2.8) and the boundary conditions (2.9) are invariant under the transformations in (3.1) subject to the conditions in (3.5). 


\subsection{Similarity Transformations}

Using the relationships in (3.5), we have from (3.1)

$$
\begin{array}{ccc}
x^{*}=e^{\varepsilon(7-5 n) / 4 \alpha_{8}} x, \quad y^{*}=e^{\varepsilon(1-n) / 2 \alpha_{8}} y, \quad \psi^{*}=e^{\varepsilon(5-3 n) / 4 \alpha_{8}} \psi, \quad \theta^{*}=\theta, \quad \phi=\phi, \\
B^{*}=e^{\varepsilon(n-1) / 2 \alpha_{8}} B, \quad \Delta T^{*}=e^{\varepsilon \alpha_{8}} \Delta T, \quad \Delta C^{*}=e^{\varepsilon \alpha_{8}} \Delta C, \quad U^{*}=e^{\varepsilon(3-n) / 4 \alpha_{8}} U, \\
v_{w}^{*}=e^{\varepsilon \alpha_{8}(n-1) / 2} v_{w}, \quad h_{f}^{*}=e^{\varepsilon \alpha_{8}(n-1) / 2} h_{f} .
\end{array}
$$

Expanding each of the transformations in (3.6) in powers of $\varepsilon$, keeping the first order term and neglecting higher order terms, we get the following characteristic equations:

$$
\begin{aligned}
\frac{4 d x}{(7-5 n) x} & =\frac{2 d y}{(1-n) y}=\frac{4 d \psi}{(5-3 n) \psi}=\frac{d \theta}{0}=\frac{d \phi}{0}=\frac{2 d B}{(n-1) B} \\
& =\frac{d(\Delta T)}{\Delta T}=\frac{d(\Delta C)}{\Delta C}=\frac{4 d U}{(n-3) U}=\frac{2 d v_{w}}{(n-1) v_{w}}=\frac{2 d h_{f}}{(n-1) h_{f}} .
\end{aligned}
$$

Solving the system of first order linear differential equations in (3.7), we get

$$
\begin{gathered}
\eta^{*}=\beta_{1} y x^{(2 n-2) /(7-5 n)}, \quad \psi^{*}=\beta_{2} x^{(5-3 n) /(7-5 n)} f(\eta), \\
\theta^{*}=\theta, \quad \phi^{*}=\phi, \quad B^{*}=\beta_{3} x^{(2 n-2) /(7-5 n)}, \\
\Delta T^{*}=\beta_{4} x^{4 /(7-5 n)}, \quad \Delta C^{*}=\beta_{5} x^{4 /(7-5 n)}, \quad U^{*}=\beta_{6} x^{(3-n) /(7-5 n),} \\
v_{w}^{*}=\beta_{7} x^{(2 n-2) /(7-5 n)}, \quad h_{f}^{*}=\beta_{8} x^{(2 n-2) /(7-5 n)},
\end{gathered}
$$

where $\beta_{i}(i=1,2,3, \ldots 8)$ are real constants. To get dimensionless form of the transformations, we define the following dimensionless transformations:

$$
\begin{gathered}
\eta=\sqrt{\operatorname{Re}} \frac{y}{L}\left(\frac{x}{L}\right)^{(2 n-2) /(7-5 n)}, \quad \psi=v \sqrt{\operatorname{Re}}\left(\frac{x}{L}\right)^{(5-3 n) /(7-5 n)} f(\eta), \\
\theta=\theta(\eta), \quad \phi=\phi(\eta), \\
B=B_{0}\left(\frac{x}{L}\right)^{(2 n-2) /(7-5 n)}, \quad \Delta T=(\Delta T)_{0}\left(\frac{x}{L}\right)^{4 /(7-5 n)}, \quad \Delta C=(\Delta C)_{0}\left(\frac{x}{L}\right)^{4 /(7-5 n)}, \\
U=U_{0}\left(\frac{x}{L}\right)^{(3-n) /(7-5 n)}, \quad v_{w}=v_{0}\left(\frac{x}{L}\right)^{(2 n-2) /(7-5 n)}, \quad h_{f}(x)=h_{0}\left(\frac{x}{L}\right)^{(2 n-2) /(7-5 n)},
\end{gathered}
$$

where $f(\eta), \theta(\eta)$ and $\phi(\eta)$, represent dimensionless velocity, temperature, and concentration functions, respectively. $B_{0},(\Delta T)_{0},(\Delta C)_{0}, U_{0}, v_{0}$ and $h_{0}$ are constants. 
Using (3.9), (2.8) become

$$
\begin{aligned}
f^{\prime \prime \prime \prime}+ & \frac{5-3 n}{7-5 n} f f^{\prime \prime \prime}-\frac{n+1}{7-5 n} f^{\prime} f^{\prime \prime}-M f^{\prime}-\lambda_{1}\left[\frac{2 n-2}{7-5 n} \eta \theta^{\prime}+\frac{4}{7-5 n} \theta\right] \\
& -\lambda_{2}\left[\frac{2 n-2}{7-5 n} \eta \phi^{\prime}+\frac{4}{7-5 n} \phi\right]=0, \\
\operatorname{Pr}^{-1} \theta^{\prime \prime} & +\frac{5-3 n}{7-5 n} f \theta^{\prime}-\frac{4}{7-5 n} f^{\prime} \theta=0, \\
\mathrm{Sc}^{-1} \phi^{\prime \prime} & +\frac{5-3 n}{7-5 n} f \phi^{\prime}-\frac{4}{7-5 n} f^{\prime} \phi-K \phi^{n}=0,
\end{aligned}
$$

subject to the boundary conditions

$$
\begin{gathered}
f(0)=\frac{7-5 n}{5-3 n} f w, \quad f^{\prime}(0)=1+a f^{\prime \prime}(0), \quad \theta^{\prime}(0)=-r[1-\theta(0)], \quad \phi(0)=1, \\
f(\infty)=f^{\prime}(\infty)=\theta(\infty)=\phi(\infty)=0,
\end{gathered}
$$

where $f w=v_{0} L / v \sqrt{\operatorname{Re}}$ is the suction/injection parameter, $\gamma=L h_{0} / \operatorname{Re} k$ is the convective heat transfer parameter, $\lambda_{1}=\mathrm{Gr} / \operatorname{Re}^{2.5}$ is the thermal buoyancy parameter, $\lambda_{2}=\mathrm{Gc} / \operatorname{Re}^{2.5}$ is the concentration buoyancy parameter, $M=\sigma B_{0}^{2} L^{2} / \rho v$ Re is the magnetic parameter, $K=L^{2} k_{0}(\Delta C)_{0}^{n-1} / v$ Re is the reaction parameter, Gr $=g \beta_{c}(\Delta T)_{0} L^{3} / v^{2}$ is the thermal Grashof number, Gc $=g \beta_{c}(\Delta T)_{0} L^{3} / v^{2}$ is the solutal Grashof number, and $a=v \sqrt{\operatorname{Re}} N_{0} / L$ is the velocity slip parameter. Note that similarity solutions will exist if $N_{1}(x)=$ $N_{0} x^{(2-2 n) /(7-5 n)}, N_{0}$ is a constant.

The physical quantities of interest are the local skin friction coefficient $C_{f x}$, local Nusselt number $\mathrm{Nu}_{x}$ (rate of heat transfer), and local Sherwood number $\mathrm{Sh}_{x}$ (rate of mass transfer), which are defined as

$$
C_{f x}=\frac{-\tau_{W}}{\rho U^{2}}, \quad \mathrm{Nu}_{x}=\frac{-x q_{w}}{k \Delta T^{\prime}}, \quad \mathrm{Sh}_{x}=\frac{-m_{w} x}{D \Delta C}
$$

where $\tau_{w}$ is the wall shear stress, $q_{w}$ is the wall heat flux, $m_{w}$ is the quantity of mass transfer through the unit area of the surface, which are given by

$$
\tau_{w}=\mu\left(\frac{\partial u}{\partial y}\right)_{y=0}, \quad q_{w}=k\left(\frac{\partial T}{\partial y}\right)_{y=0}, \quad m_{w}=D\left(\frac{\partial C}{\partial y}\right)_{y=0} .
$$

It can be shown that physical quantities are proportional to $-f^{\prime \prime}(0),-\theta^{\prime}(0)$ and $-\phi^{\prime}(0)$.

\section{Results and Discussion}

Equations (3.10) with boundary conditions (3.11) were solved using the dsolve command in MAPLE 14 with numeric option. Depending upon the nature of the ordinary differential 
Table 1: Comparison of results for $f^{\prime \prime}(0)$ and $-\phi^{\prime}(0)$ for different values of parameters.

\begin{tabular}{|c|c|c|c|c|}
\hline \multicolumn{5}{|c|}{$\operatorname{Pr}=0.72, \operatorname{Pr}=0.72, \lambda_{1}=1, \lambda_{2}=1, K=1$} \\
\hline \multirow{2}{*}{ Sc } & \multicolumn{2}{|c|}{ Fan et al. [17] } & \multicolumn{2}{|c|}{ Present results } \\
\hline & $f^{\prime \prime}(0)$ & $\phi^{\prime}(0)$ & $f^{\prime \prime}(0)$ & $-\phi^{\prime}(0)$ \\
\hline 0.1 & 0.9098 & 0.13678 & 0.9099 & 0.1368 \\
\hline 0.72 & 0.47511 & 0.28697 & 0.4751 & 0.287 \\
\hline 5 & 0.23157 & 0.77288 & 0.2316 & 0.7729 \\
\hline \multicolumn{5}{|c|}{$\operatorname{Pr}=0.72, \operatorname{Pr}=0.72, \mathrm{Sc}=0.72, \lambda_{2}=1, K=1$} \\
\hline$\lambda_{1}$ & \multicolumn{2}{|c|}{ Fan et al. [17] } & \multicolumn{2}{|c|}{ Present results } \\
\hline 0 & 0.06194 & 0.29757 & 0.0619 & 0.2976 \\
\hline 0.4 & 0.24155 & 0.29252 & 0.2415 & 0.2925 \\
\hline 1 & 0.47511 & 0.28697 & 0.4751 & 0.287 \\
\hline \multicolumn{5}{|c|}{$\operatorname{Pr}=0.72, \operatorname{Pr}=0.72, \lambda_{1}=1, \mathrm{Sc}=0.72, K=1$} \\
\hline$\lambda_{2}$ & \multicolumn{2}{|c|}{ Fan et al. [17] } & \multicolumn{2}{|c|}{ Present results } \\
\hline 0 & 0.14425 & 0.29487 & 0.1442 & 0.2949 \\
\hline 0.4 & 0.2835 & 0.29133 & 0.2835 & 0.2913 \\
\hline 1 & 0.47511 & 0.28697 & 0.4751 & 0.287 \\
\hline \multicolumn{5}{|c|}{$\operatorname{Pr}=0.72, \operatorname{Pr}=0.72, \lambda_{1}=1, \lambda_{2}=1, \mathrm{Sc}=0.72$} \\
\hline$K$ & \multicolumn{2}{|c|}{ Fan et al. [17] } & \multicolumn{2}{|c|}{ Present results } \\
\hline 0 & 0.53159 & 0.00015 & 0.5316 & 0.00015 \\
\hline 1 & 0.47511 & 0.28697 & 0.4751 & 0.287 \\
\hline 3 & 0.42378 & 0.61989 & 0.4238 & 0.6199 \\
\hline
\end{tabular}

equations, MAPLE uses a suitable scheme based on trapezoid or midpoint rule with Richardson extrapolation or deferred correction enhancement. In order to justify the accuracy of our numerical method, we compared our results with available data in Table 1 and found a good agreement. The influence of different parameters on the dimensionless velocity, temperature, and concentration is shown in Figures 2, 3, and 4, respectively. Figure 2(a) exhibits the influence of thermal buoyancy parameter $\lambda_{1}$ and suction/injection parameter $f w$, whilst Figure 2(b) shows the effects of solutal buoyancy parameter $\lambda_{2}$ and suction/injection parameter $f w$, on the dimensionless fluid velocity $f^{\prime}(\eta)$ profiles. We further observed that velocity increases with buoyancy parameters $\lambda_{1}$ and $\lambda_{2}$. Figure 2(c) describes the influence of magnetic field parameter $M$ on velocity $f^{\prime}(\eta)$ profiles. It is found that as $M$ increases, the fluid velocity decreases. The effect of velocity slip parameter on the dimensionless velocity is depicted in Figure 2(d). It is observed that an increase in the velocity slip reduces the dimensionless velocity at the surface. In all cases suction increases the velocity whilst injection decreases the velocity, as expected.

Figures 3(a), 3(b), 3(c), and 3(d) display effects of various parameters on the dimensionless temperature function. The dimensionless temperature decreases with suction and increases with injection, as expected. Note that as we increase the values of $\lambda_{1}$ and $\lambda_{2}$, the values of $\theta(\eta)$ decrease (Figures 3(a) and 3(b)). It is found that magnetic field increases temperature whilst Prandtl number decreases the temperature. Figures 4(a), 4(b), 4(c), and 4(d) exhibit influence of suction/injection $f w$, buoyancies $\lambda_{1}, \lambda_{2}$, the magnetic field $M$ and the Schmidt number Sc on the dimensionless concentration $\phi(\eta)$ function. The dimensionless concentration decreases with the increase of $f w$. We also notice from Figures 4(a) and $4(\mathrm{~b})$ as we increase the values of $\lambda_{1}$ and $\lambda_{2}$, values of $\phi(\eta)$ decrease. Figure 4(c) shows that 


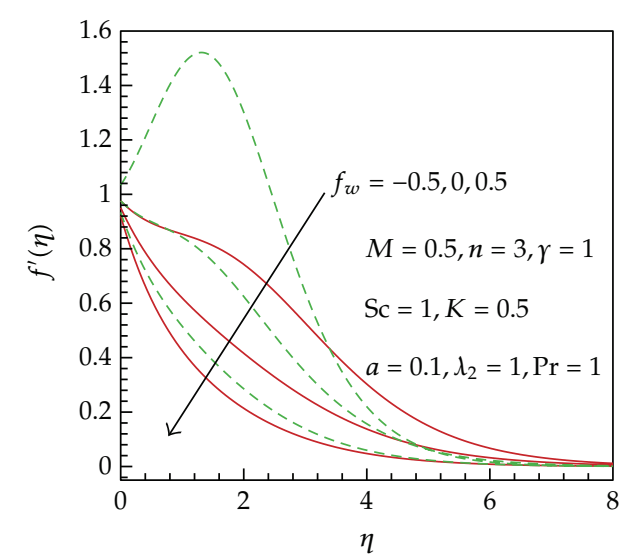

$\lambda_{1}$

$-0.1$

$---1$

(a)

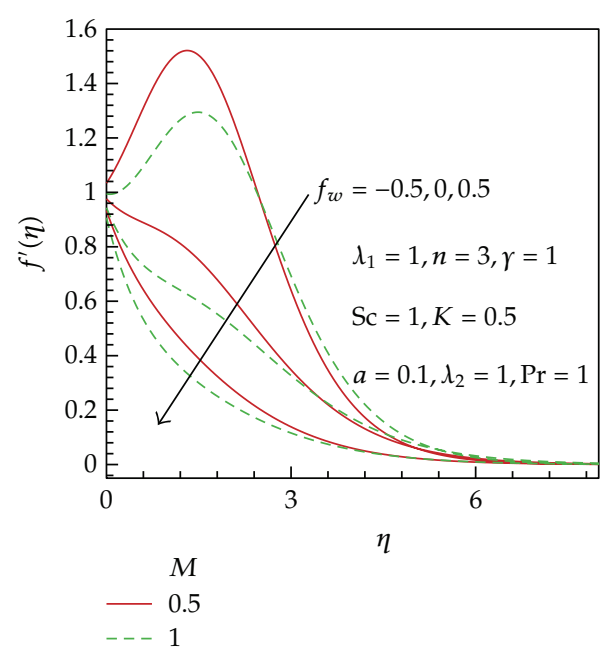

(c)

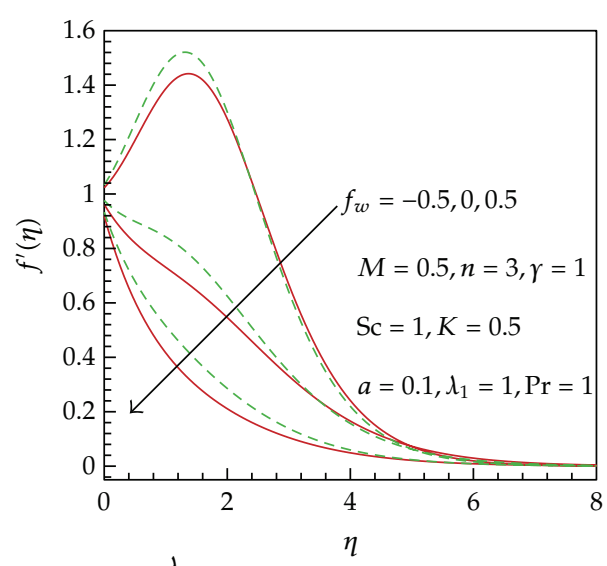

$\lambda_{2}$

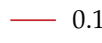

$--1$

(b)

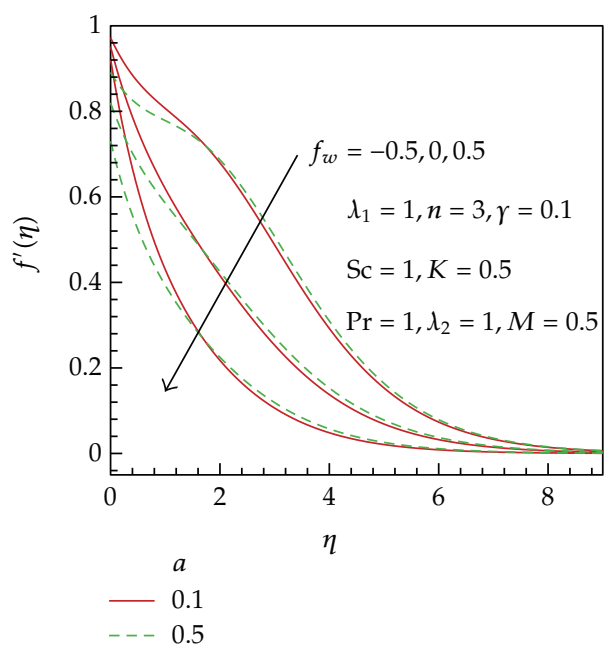

(d)

Figure 2: Effects of different parameters on the dimensionless velocity.

the increasing values of magnetic field parameter lead to increase the concentration whilst Figure 4(d) shows that increasing values of Schmidt number Sc leads to decrease the concentration, as expected.

Figures 5(a) and 5(b) depict the variation of the buoyancies $\lambda_{1}, \lambda_{2}$, the slip $a$ and the suction/injection $f w$ parameters on the dimensionless shear stress. It is observed that suction enhanced the values of the dimensionless shear stress. We also see that the velocity slip $a$ and the parameters $\lambda_{1}, \lambda_{2}$ increase the values of the dimensionless shear stress fall. The effect of Prandtl number Pr, convective heat transfer parameter $\gamma$, order of chemical reaction $n$, and the suction/injection parameter $f w$ on the dimensionless rate of heat transfer is shown in Figures 6(a) and 6(b). From Figure 6(a) we can conclude that as $\operatorname{Pr}, \gamma$ and $f w$ increase, the values of the dimensionless rate of heat transfer increase. An increase in the order of reaction 

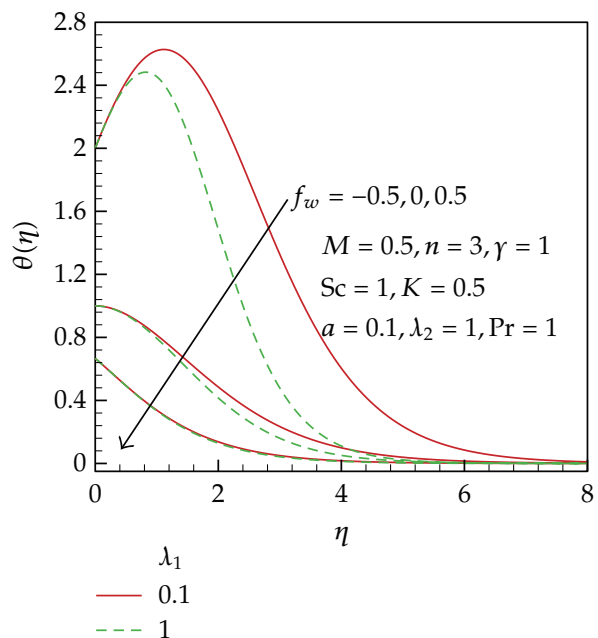

$---1$

(a)

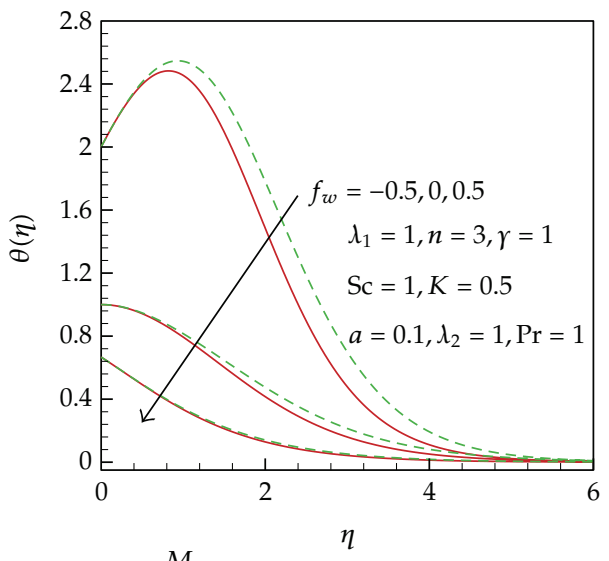

$M$
-0.5

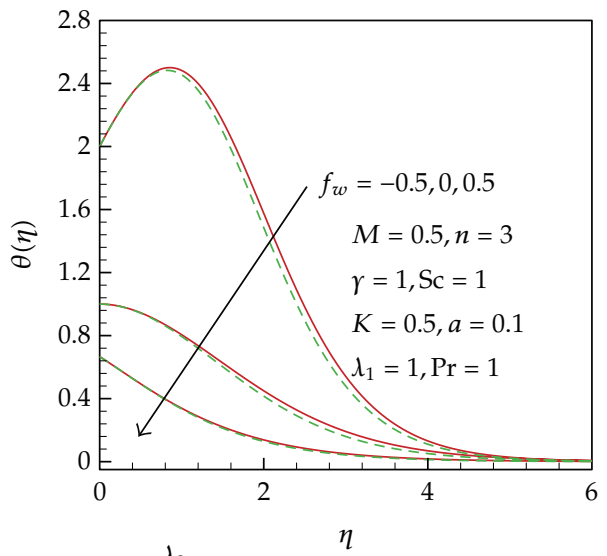

$\lambda_{2}$

-0.1
--1

(b)

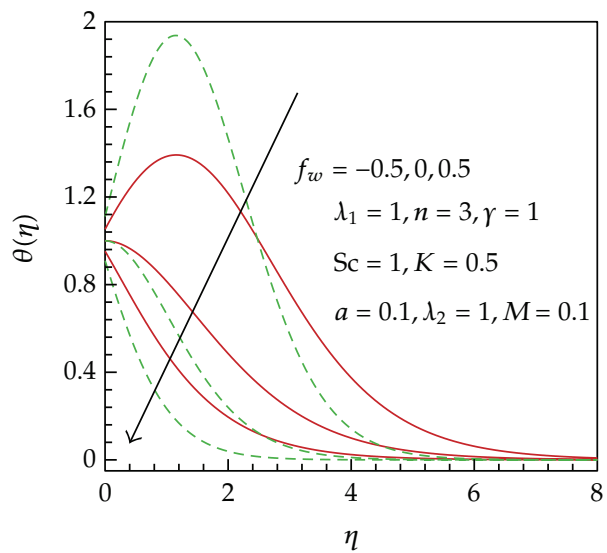

$\operatorname{Pr}$
$-\quad 1$
---2

(c)

(d)

Figure 3: Effects of different parameters on the dimensionless temperature.

causes decrease in the dimensionless rate of heat transfer (Figure 6(b)). The variation of the dimensionless rate of mass transfer with different parameters is shown in Figures 7(a) and $7(b)$. Like the dimensionless rate of heat transfer, the dimensionless rate of mass transfer increases with an increase in Sc, $K$, and $f w$ (Figure 7(a)) and decreases with an increase in the order of reaction $n$.

\section{Conclusions}

MHD boundary layer equations for mixed convection of Newtonian fluids along a moving horizontal plate with velocity slip and thermal convective boundary condition are 

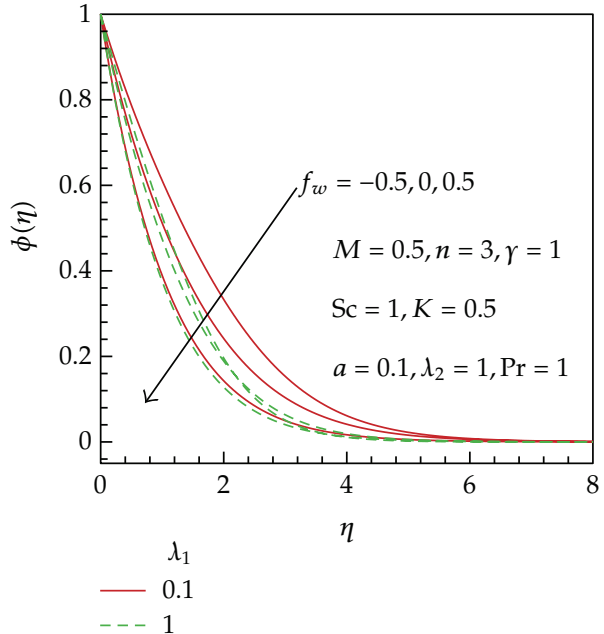

(a)

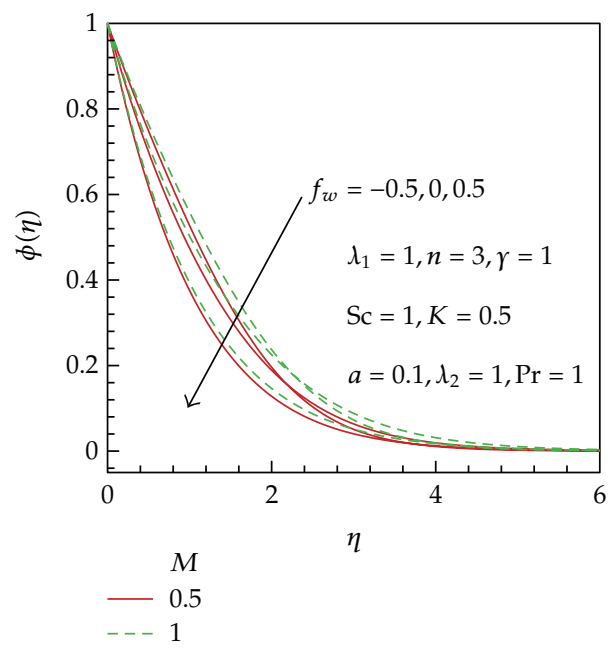

(c)
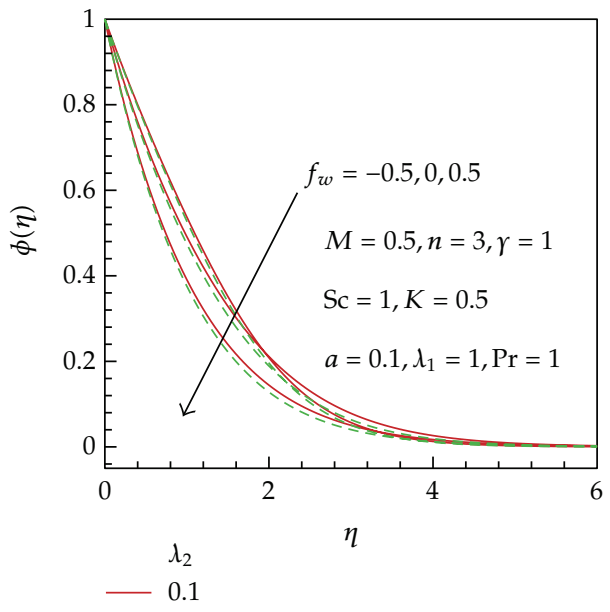

$--1$

(b)

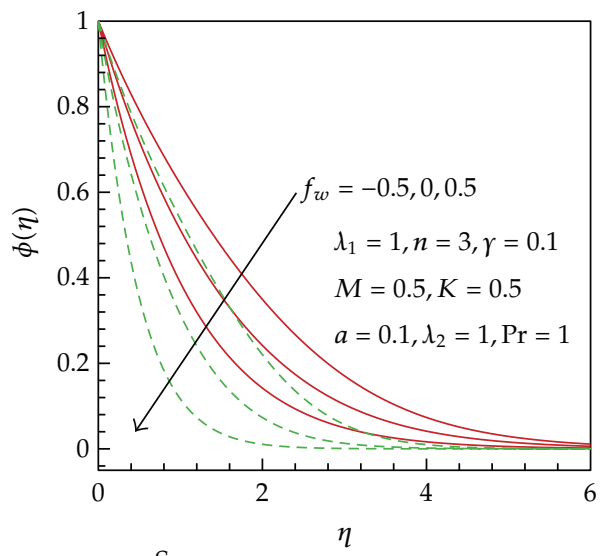

Sc
$-\quad 1$
---3

Figure 4: Effects of different parameters on the dimensionless concentration.

transformed into similarity equations using one-parameter continuous group method and then solved numerically using an implicit finite difference numerical method. Our analysis revealed that $-f^{\prime \prime}(0)$ increases with suction $f w$ and decreases with $a, \lambda_{1}$, and $\lambda_{2}$ whilst the values of $-\theta^{\prime}(0)$ enhance with $f w$, Pr, and $\gamma$. The values of $-\phi^{\prime}(0)$ enhance with $f w$, Sc and $k$.

\section{Nomenclature}

a, $B(x)$ : Velocity slip parameter, variable magnetic field

$C, c_{p}$ : Concentration, specific heat at constant pressure

$c_{f x}$ : $\quad$ Skin friction factor

$D, f: \quad$ Mass diffusivity, dimensionless stream function 


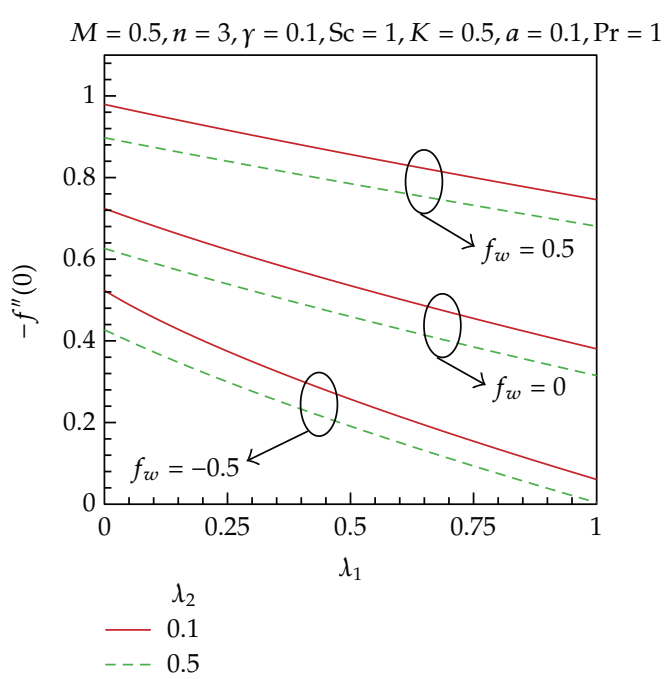

(a)

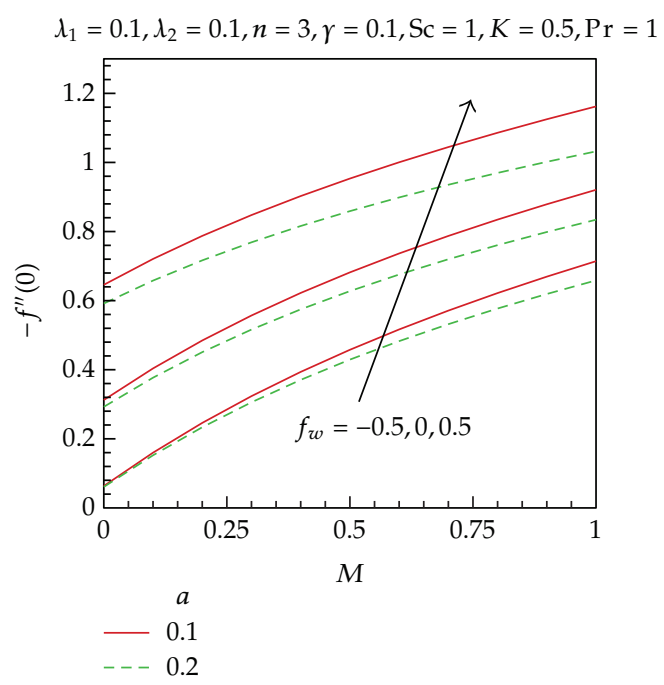

(b)

Figure 5: Effects of different parameters on the dimensionless shear stress.

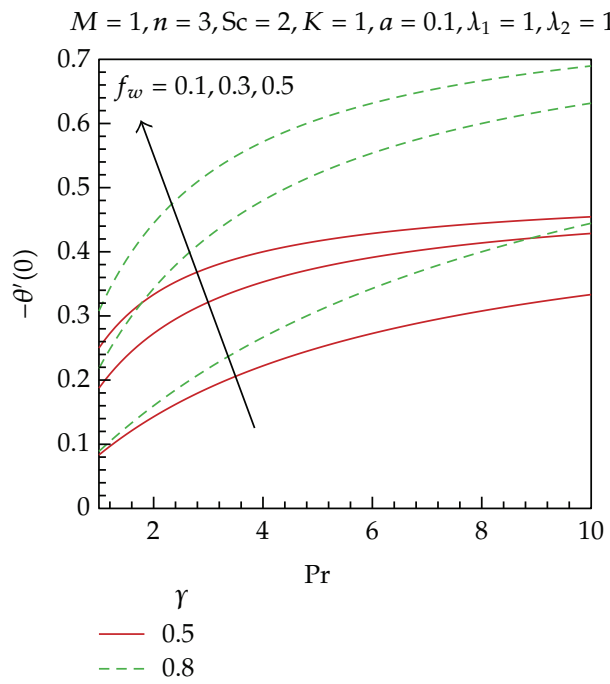

(a)

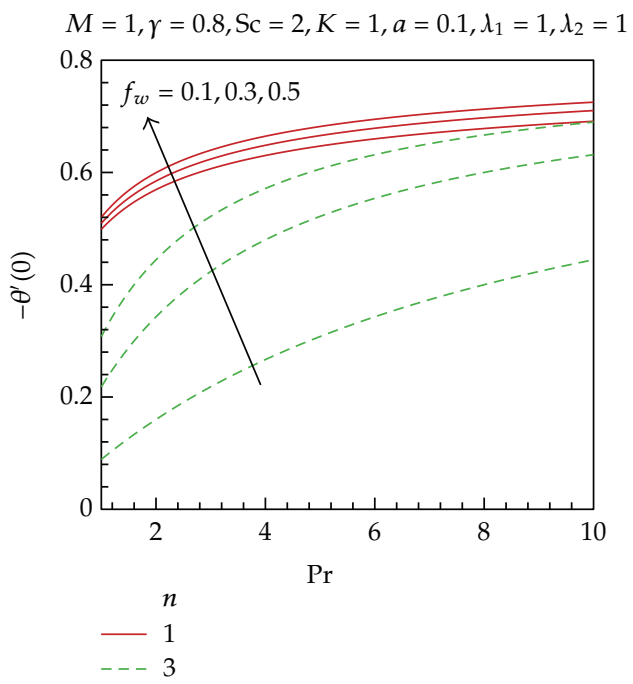

(b)

Figure 6: Effects of different parameters on the dimensionless rate of heat transfer.

$f w, g:$ Suction/injection parameter, gravitational acceleration

Gr, Gc: Thermal and solutal Grashof number

$h_{f}(x)$ : Heat transfer coefficient

$k, k_{0}$ : Thermal conductivity, reaction rate constant

$K, L$ : Chemical reaction parameter, characteristic length of the plate 


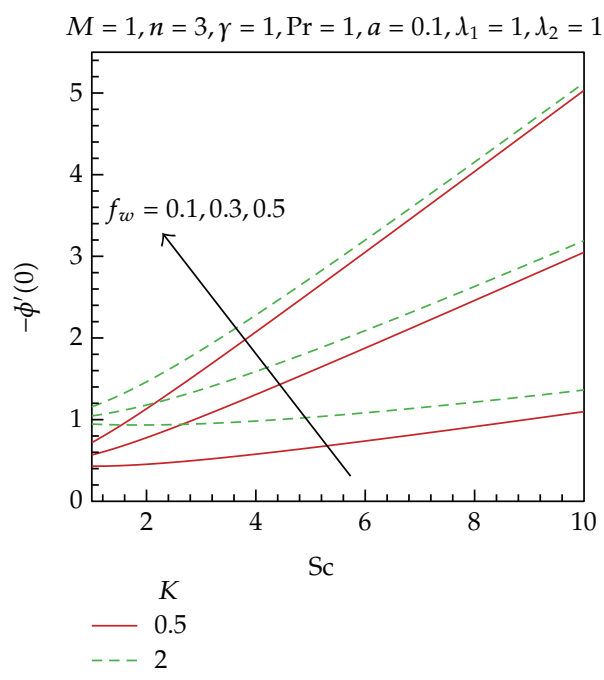

(a)

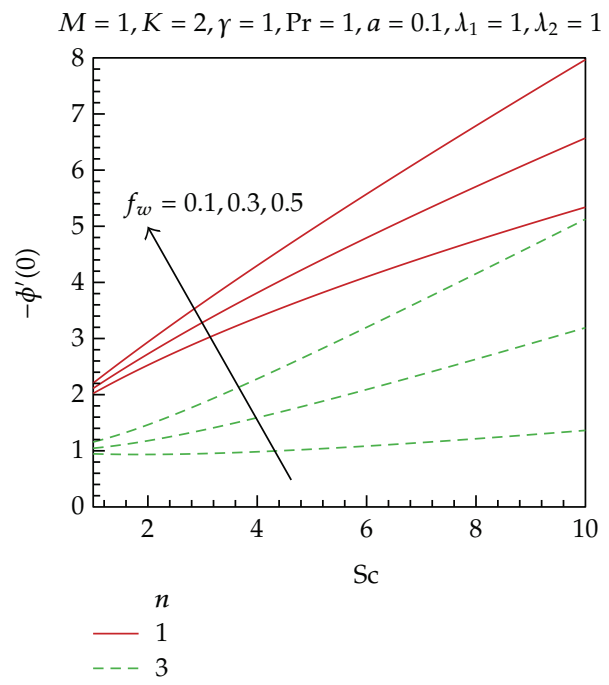

(b)

Figure 7: Effects of different parameters on the dimensionless rate of mass transfer.

$M, n, \mathrm{Nu}_{x}$ : Magnetic parameter, order of chemical reaction, Nusselt number

$N_{1}(x), p: \quad$ Variable slip factor

Pr, Re, Sc: Prandtl, Reynolds, and Schmidt number

$\mathrm{Sh}_{x} T: \quad$ Sherwood number, dimensional temperature

$U, U_{0}$ : Velocity of the plate and reference velocity

$u, v: \quad$ Velocity components in $x$-direction and $y$-direction

$x, y: \quad$ Coordinate along and normal to plate.

\section{Greek Symbols}

$\alpha_{i}, \beta_{i}$ : Real numbers

$\beta_{T}, \beta_{C}$ : Coefficient of thermal and mass expansion

$\gamma, \Gamma$ : Convective heat transfer parameter, group

$\mu, v$ : Dynamic and kinematic coefficient of viscosity

$\varepsilon, \sigma: \quad$ Parameter of the group transformations, electric conductivity

$\lambda_{1}, \lambda_{2}$ : Thermal, concentration buoyancy parameter

$\eta, \psi:$ Similarity independent variable, stream function

$\theta, \phi$ : Dimensionless temperature and concentration function

$\rho: \quad$ Density of the fluid.

\section{Subscript}

$\infty:$ Condition at infinity

$w$ : Condition at the wall. 


\section{References}

[1] T. K. Sengupta, S. Unnikrishnan, S. Bhaumik, P. Singh, and S. Usman, "Linear spatial stability analysis of mixed convection boundary layer over a heated plate," Progress in Applied Mathematics, vol. 1, pp. 71-89, 2011.

[2] F. M. Ali, R. Nazar, N. M. Arifin, and I. Pop, "MHD mixed convection boundary layer flow toward a stagnation point on a vertical surface with induced magnetic field," Journal of Heat Transfer, vol. 133, no. 2, Article ID 022502, pp. 1-6, 2011.

[3] S. V. Subhashini, N. Samuel, and I. Pop, "Effects of buoyancy assisting and opposing flows on mixed convection boundary layer flow over a permeable vertical surface," International Communications in Heat and Mass Transfer, vol. 38, no. 4, pp. 499-503, 2011.

[4] F. P. Incropera, "Buoyancy effects in double-diffusive mixed convection Flows," in Proceedings of the 8th International Heat Transfer Conference, C. L. Tein, V. P. Carey, and J. K. Ferrel, Eds., pp. 121-130.

[5] T. S. Chen and B. S. Armaly, "Mixed convection in external flow," in Handbook of Single-Phase Convective Heat Transfer, S. Kakac, R. K. Shah, and W. Aung, Eds., Wiley, New York, NY, USA, 1987.

[6] Y. Jaluria, "Basic of Natural ConvectionS," in Handbook of Single-Phase Convective Heat Transfer, Kakac, R. K. Shah, and W. Aung, Eds., Wiley, New York, NY, USA, 1987.

[7] B. Gebhart, Y. Jaluria, R. L. Mahajan, and B. Sammarkia, Buoyancy-Induced Flows and Transport, Chapter 10, Hemisphere, Washington, DC, USA, 1988.

[8] A. Bejan, Convection Heat Transfer, Wiley, New York, NY, USA, 1995.

[9] I. Pop and D. B. Ingham, Convective Heat Transfer, Pergamon, Amsterdam, The Netherlands, 2001.

[10] J. R. Lloyd and E. M. Sparrow, "Combined forced and free convection flow on vertical surfaces," International Journal of Heat and Mass Transfer, vol. 13, no. 2, pp. 434-438, 1970.

[11] W. Schneider, "A similarity solution for combined forced and free convection flow over a horizontal plate," International Journal of Heat and Mass Transfer, vol. 22, no. 10, pp. 1401-1406, 1979.

[12] G. Wilks, "Combined forced and free convection flow on vertical surfaces," International Journal of Heat and Mass Transfer, vol. 16, no. 10, pp. 1958-1964, 1973.

[13] L. S. Yao, "Two-dimensional mixed convection along a flat plate," Journal of Heat Transfer, vol. 109, no. 2, pp. 440-445, 1987.

[14] N. Ramachandran, T. S. Chen, and B. F. Armaly, "Mixed convection in stagnation flows adjacent to vertical surfaces," Journal of Heat Transfer, vol. 110, no. 2, pp. 373-377, 1988.

[15] G. S. Moulic and L. S. Yao, "Mixed convection along a wavy surface," ASME Journal of Heat Transfer, vol. 111, pp. 974-979, 1989.

[16] J. Daskalakis, N. Kafoussias, A. Lewkowicz, and E. W. Williams, "Similarity solution for free and forced convection hydromagnetic flow over a horizontal semi-infinite plate through a non-homogeneous porous medium," Astrophysics and Space Science, vol. 151, no. 2, pp. 217-226, 1989.

[17] J. R. Fan, J. M. Shi, and X. Z. Xu, "Similarity solution of mixed convection over a horizontal moving plate," Heat and Mass Transfer, vol. 32, no. 3, pp. 199-206, 1997.

[18] E. Magyari, I. Pop, and B. Keller, "Mixed convection boundary-layer flow past a horizontal permeable flat plate," Fluid Dynamics Research, vol. 31, no. 3, pp. 215-225, 2002.

[19] I. A. Hassanien, A. H. Essawy, and N. M. Moursy, "Variable viscosity and thermal conductivity effects on combined heat and mass transfer in mixed convection over a UHF/UMF wedge in porous media: the entire regime," Applied Mathematics and Computation, vol. 145, no. 2-3, pp. 667-682, 2003.

[20] O. Aydin and A. Kaya, "Mixed convection of a viscous dissipating fluid about a vertical flat plate," Applied Mathematical Modelling, vol. 31, no. 5, pp. 843-853, 2007.

[21] A. Ishak, "Mixed convection boundary layer flow over a horizontal plate with thermal radiation," Heat and Mass Transfer, vol. 46, no. 2, pp. 147-151, 2009.

[22] S. G. Moulic and L. S. Yao, "Mixed convection along a semi-infinite vertical flat plate with uniform surface heat flux," Journal of Heat Transfer, vol. 131, no. 2, pp. 1-8, 2009.

[23] P. Datta, S. V. Subhashini, and R. Ravindran, "Influence of surface mass transfer on mixed convection flows over non-isothermal horizontal flat plates," Applied Mathematical Modelling, vol. 33, no. 3, pp. 1285-1294, 2009.

[24] A. Ishak, R. Nazar, N. Bachok, and I. Pop, "MHD mixed convection flow near the stagnation-point on a vertical permeable surface," Physica A, vol. 389, no. 1, pp. 40-46, 2010.

[25] E. Magyari, "Comment on "Mixed convection boundary layer flow over a horizontal plate with thermal radiation" by A. Ishak, Heat Mass Transfer," Heat and Mass Transfer, vol. 46, no. 8-9, pp. 809-810, 2010. 
[26] L. M. Jiji, Heat Convection, cshapter 11, Springer, 2nd edition, 2009.

[27] G. M. Hak, "Flow physics in the MEMS handbook," in MEMS Handbook, M. Gad-el-Hak, Ed., chapter 4, CRC Press, Boca Raton, Fla, USA, 2002.

[28] A. Aziz, "Hydrodynamic and thermal slip flow boundary layers over a flat plate with constant heat flux boundary condition," Communications in Nonlinear Science and Numerical Simulation, vol. 15, no. 3, pp. 573-580, 2010.

[29] A. Yoshimura and R. K. Prud'homme, "Wall slip corrections for Couette and parallel disk viscometers," Journal of Rheology, vol. 32, no. 1, pp. 53-67, 1988.

[30] M. M. Rahman, "Locally similar solutions for hydromagnetic and thermal slip flow boundary layers over a flat plate with variable fluid properties and convective surface boundary condition," Meccanica, vol. 46, no. 5, pp. 1127-1143, 2011.

[31] G. P. Duncan and G. P. Peterson, "Review of Microscale Heat Transfer," Applied Mechanics Review, vol. 47, pp. 397-428, 1994.

[32] M. Gad-el-Hak, "Fluid mechanics of microdevices-the freeman scholar lecture," ASME Journal of Fluids Engineering, vol. 121, no. 1, pp. 5-33, 1999.

[33] T. Fang and C. F. Lee, "A moving-wall boundary layer flow of a slightly rarefied gas free stream over a moving flat plate," Applied Mathematics Letters, vol. 18, no. 5, pp. 487-495, 2005.

[34] M. J. Martin and I. D. Boyd, "Falkner-Skan flow over a wedge with slip boundary conditions," Journal of Thermophysics and Heat Transfer, vol. 24, no. 2, pp. 263-270, 2010.

[35] M. T. Matthews and J. M. Hill, "Micro/nano thermal boundary layer equations with slip-creep-jump boundary conditions," IMA Journal of Applied Mathematics, vol. 72, no. 6, pp. 894-911, 2007.

[36] C. Y. Wang, "Analysis of viscous flow due to a stretching sheet with surface slip and suction," Nonlinear Analysis. Real World Applications, vol. 10, no. 1, pp. 375-380, 2009.

[37] K. Cao and J. Baker, "Slip effects on mixed convective flow and heat transfer from a vertical plate," International Journal of Heat and Mass Transfer, vol. 52, no. 15-16, pp. 3829-3841, 2009.

[38] W. F. Ames, Nonlinear Partial Differential Equations in Engineering, Academic Press, New York, NY, USA, 1965.

[39] W. E. Boyce, Elementary Differential Equations and Boundary Value Problems, John Wiley and Sons, New York, NY, USA, 1977.

[40] T. Y. Na, Computational methods in engineering boundary value problems, vol. 145 of Mathematics in Science and Engineering, Academic Press, New York, NY, USA, 1979.

[41] E. Zauderer, Partial Differential Equations of Applied Mathematics, Pure and Applied Mathematics, John Wiley \& Sons, New York, NY, USA, 1983.

[42] R. Seshadri and T. Y. Na, Group Invariance in Engineering Boundary Value Problems, Springer, New York, NY, USA, 1985.

[43] D. Kincaid and W. Cheney, Numerical Analysis, Brooks/Cole Publishing, Pacific Grove, Calif, USA, 1991.

[44] W. A. Strauss, Partial Differential Equations. An Introduction, John Wiley \& Sons, New York, NY, USA, 1992.

[45] A. Aziz, Heat Conduction with Maple, R.T Edwards, 2005.

[46] R. E. White and V. R. Subramanian, Computational Methods InChemical Engineering With Maple, Springer, 2010.

[47] J. C. Slattery, Advanced Transport Phenomena, Cambridge University Press, Cambridge, UK, 1999.

[48] F. P. Incropera, D. P. Dewitt, T. L. Bergman, and A. S. Lavine, Fundamentals of Heat and Mass Transfer, John Wiley, New York, NY, USA, 6th edition, 2007.

[49] S. Mukhopadhyay and H. I. Andersson, "Effects of slip and heat transfer analysis of flow over an unsteady stretching surface," Heat and Mass Transfer, vol. 45, no. 11, pp. 1447-1452, 2009. 


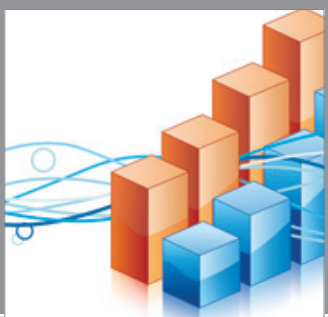

Advances in

Operations Research

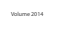

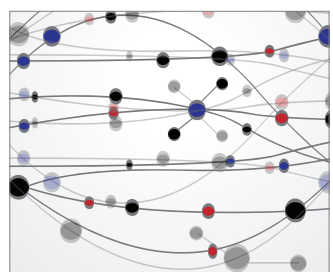

\section{The Scientific} World Journal
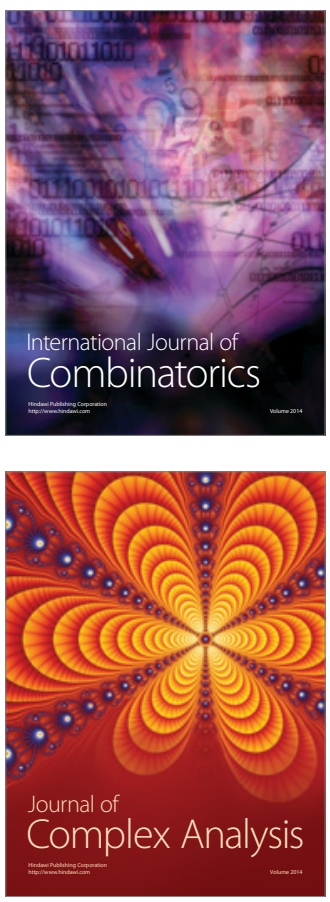

International Journal of

Mathematics and

Mathematical

Sciences
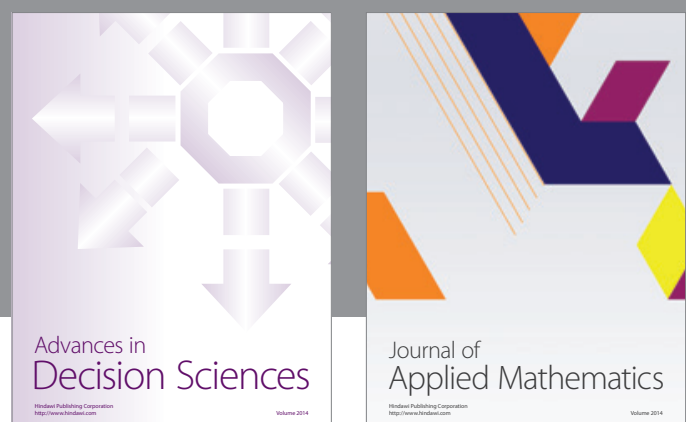

Journal of

Applied Mathematics
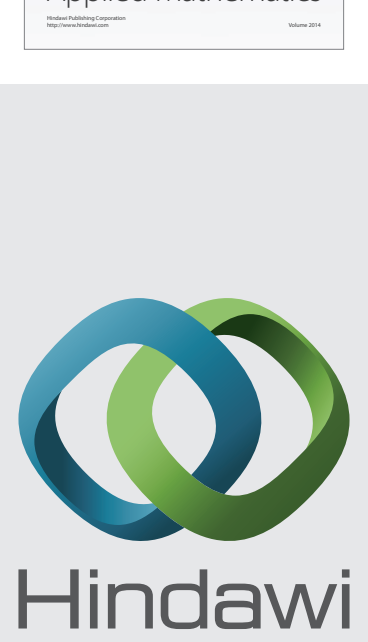

Submit your manuscripts at http://www.hindawi.com
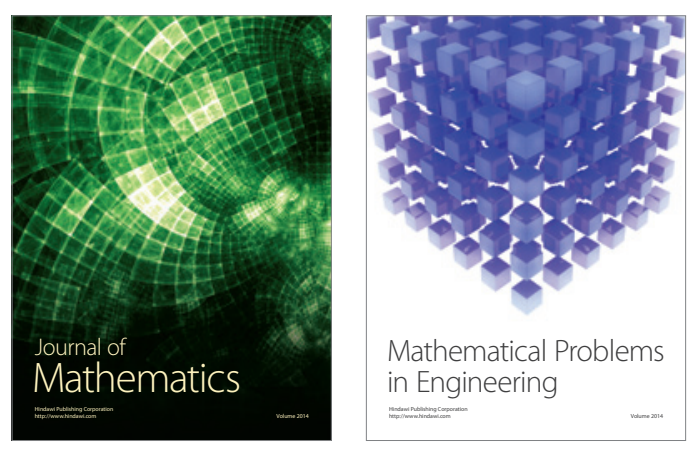

Mathematical Problems in Engineering
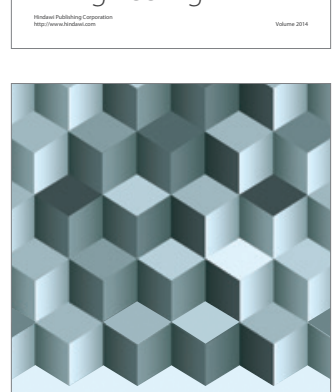

Journal of

Function Spaces
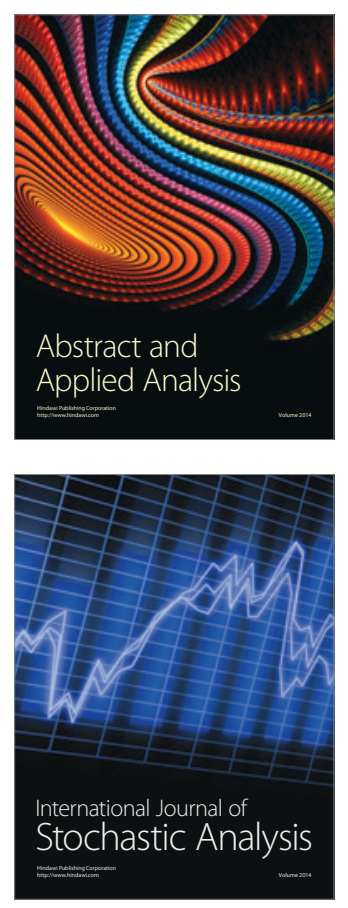

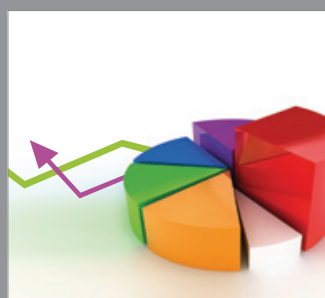

ournal of

Probability and Statistics

Promensencen
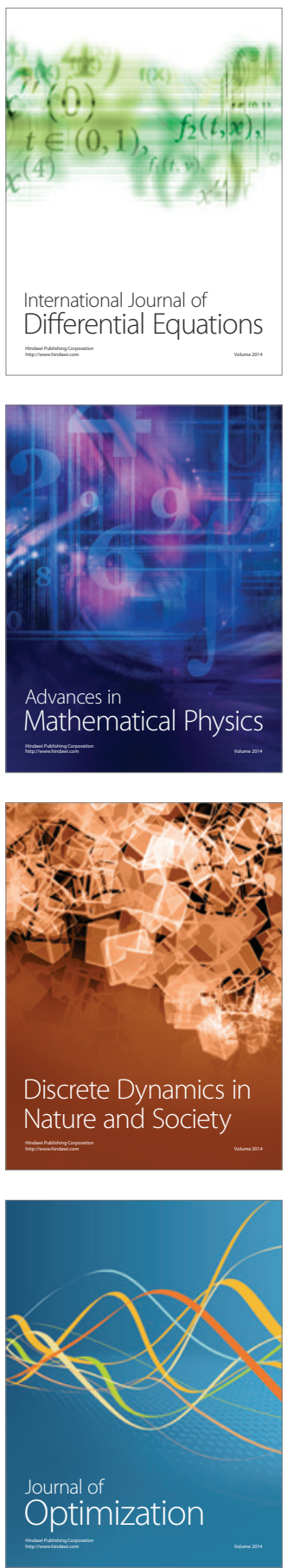